\title{
Saturation of CVD diamond detectors ${ }^{*}$
}

\author{
Lucile S. Dauffy ${ }^{1}$, Richard A. Lerche ${ }^{1}$, Greg J. Schmid ${ }^{1}$, Jeffrey A. Koch ${ }^{1}$, and \\ Christopher Silbernagel ${ }^{2}$ \\ ${ }^{1}$ Lawrence Livermore National Laboratory, Livermore, CA 94550, USA \\ ${ }^{2}$ Bechtel Nevada calibration facilities, Livermore, CA 94551, USA
}

\begin{abstract}
A 5 x 0.25 mm Chemical Vapor Deposited (CVD) diamond detector, with a voltage bias of + $250 \mathrm{~V}$, was excited by a $400 \mathrm{~nm}$ laser ( $3.1 \mathrm{eV}$ photons) in order to study the saturation of the wafer and its surrounding electronics. In a first experiment, the laser beam energy was increased from a few tens of a $\mathrm{pJ}$ to about $100 \mu \mathrm{J}$, and the signal from the diamond was recorded until full saturation of the detection system was achieved. Clear saturation of the detection system was observed at about $40 \mathrm{~V}$, which corresponds with the expected saturation at $10 \%$ of the applied bias $(250 \mathrm{~V})$. The results indicate that the interaction mechanism of the $3.1 \mathrm{eV}$ photons in the diamond $\left(\mathrm{E}_{\text {bandgap }}=5.45 \mathrm{eV}\right)$ is not a multi-photon process but is linked to the impurities and defects of the crystal. In a second experiment, the detector was irradiated by a saturating first laser pulse and then by a delayed laser pulse of equal or smaller amplitude with delays of 5, 10, and 20 ns. The results suggest that the diamond and associated electronics recover within 10 to $20 \mathrm{~ns}$ after a strong saturating pulse.
\end{abstract}

\section{Introduction}

The mission of the National Ignition Facility (NIF) is to produce high-energy-density plasmas and, ultimately, to demonstrate fusion ignition through inertial confinement. To do so, advanced nuclear diagnostics are required to measure the characteristics of an implosion including neutron yield, areal density $(\rho \mathrm{R})$, hot spot size and ion temperature $\left(\mathrm{T}_{\mathrm{i}}\right)$, fuel symmetry, and bang time. One of these diagnostics, the Chemical Vapor Deposition (CVD) diamond detector, is being tested to evaluate the feasibility of measuring areal density by determining the ratio of downscattered (scattered inside the target) to primary neutrons using time-of-flight spectroscopy [1].

In a previous study [2], CVD diamond time-of-flight signals were calculated for two typical DT implosions, a 1D clean-burning shot (successful ignition) and a 2D fizzle shot (typical failed ignition), at several distances from the target chamber center. The intensity of the $14 \mathrm{MeV}$ peak is several orders of magnitude larger than the downscattered neutron signal. This can lead to saturation of the detector and its surrounding electronics by the $14-\mathrm{MeV}$ neutrons and to a nonlinear response to the downscattered neutrons. In order to quantify this non-linearity after the large pulse, and thus the error

* This work has been performed under the auspices of U.S. Department of Energy by the University of California Lawrence Livermore National Laboratory under Contract No. W-7405-Eng-48. 
in the number of measured downscattered neutrons, the saturation of a CVD diamond detector and surrounding electronics has been studied using a $400 \mathrm{~nm}$ laser (3.1 eV photons) [3]. The detector was a $5 \mathrm{~mm}$ (diameter) x $0.25 \mathrm{~mm}$ (thickness) polycrystalline CVD diamond, coated with titanium / platinum / gold electrodes on the top and bottom surfaces. The applied bias, HV, through the CVD diamond wafer was $+250 \mathrm{~V}$ and the oscilloscope was a Tektronix 6604 (6 GHz bandwidth, $20 \mathrm{GHz}$ sampling frequency). The incident laser beam was $250 \mu \mathrm{m}$ x $5 \mathrm{~mm}$ in cross-section and its pulse length was less than $750 \mathrm{fs}$.

The experiment was divided into two measurements. The first one consisted of obtaining the saturation profile of the CVD diamond by irradiating the wafer with a laser pulse of increasing energy, and recording the output voltage. In a second measurement, the detector was irradiated by a first saturating pulse and then by a second pulse of equal or lesser intensity, delayed by 5, 10, and 20 ns.

\section{First measurement's results: signal height, width, and integral vs. incident energy}

The results of the first measurement are shown in figure 1 . The maximum pulse height, $\mathrm{V}_{\max }(\mathrm{V})$, starts to clearly depart from linearity at about $40 \mathrm{~V}$, which confirms that CVD diamonds like many semiconductors follow the " $10 \%$ rule", i.e. respond linearly below $10 \%$ of the applied voltage [4]. The right graph of this figure also shows linearity of the full width half maximum (FWHM) which stays constant $(0.45 \mathrm{~ns})$ up to about $1 \mu \mathrm{J}$, incident energy consistent with that at which $\mathrm{V}_{\max }$ clearly departs from linearity. The integral under the pulse, $\mathrm{S}(\mathrm{V} \mathrm{ns})$, which is proportional to the charge collected by the oscilloscope, departs from linearity at a higher voltage and does not seem to reach full saturation.
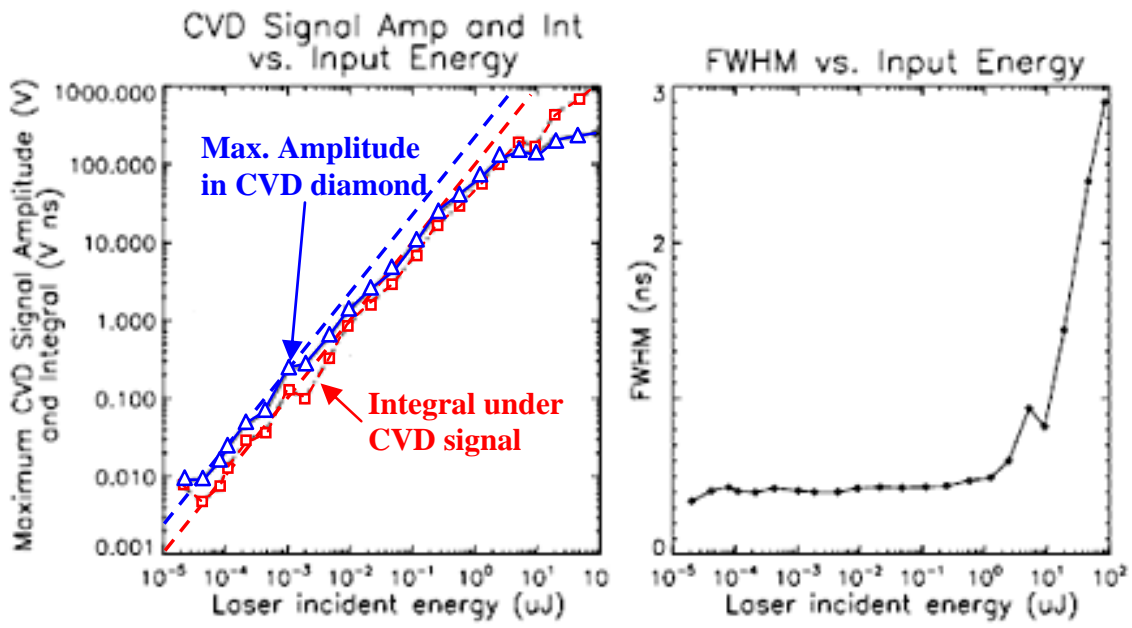

Figure 1. Maximum signal amplitude and integral under the pulse in the CVD diamond wafer versus incident energy (left graph), and the FWHM of the pulse versus input energy (right graph). The straight-dashed lines are the linearity lines.

\section{Second measurement's result: generation of two pulses delayed by 5, 10, and 20 ns}

Figure 2 presents $V_{\max }$ and $S$ versus incident energy, $E_{\text {in }}$, for the $2^{\text {nd }}$ pulse, which shows the effect of the $1^{\text {st }}$ large pulse on the detector's response and recovery. The units for $\mathrm{E}_{\mathrm{in}}$ are arbitrary but consistent. These results suggest that the detector saturates in the 5 ns delay case, i.e. the charge collected ( $\propto S$ ) over incident energy ratio is not constant, since the $2^{\text {nd }}$ pulse integral $S$ is non linear at the lowest incident energy. In both the 10 and 20 ns case, the detector appears to respond linearly for incident energies below about $10 \mathrm{~V}$. Because there are just a few sampling points, this voltage limit, $10 \mathrm{~V}$, can be said following the "10\% rule”. Charge seems to be conserved when the detector and associated electronics have at least $10 \mathrm{~ns}$ to recover. 

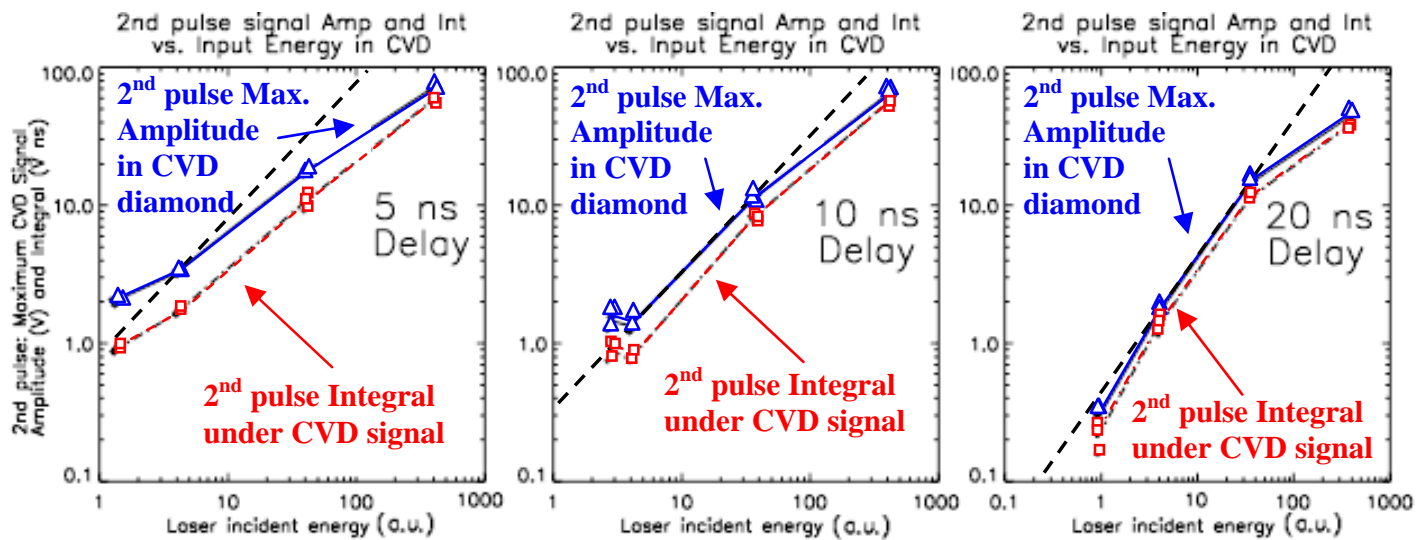

Figure 2. Maximum signal amplitude, $V_{\max }(V)$, of the $2^{\text {nd }}$ pulse and integral under the pulse, $S(V$ ns), in the CVD diamond wafer versus incident energy for the $2^{\text {nd }}$ pulse (in arbitrary but consistent units). The straightdashed lines are the linearity lines. Results are shown for the 3 delay times, 5, 10, and 20 ns in between both pulses.

\section{Conclusion}

This work used a $400 \mathrm{~nm}$ laser to study the saturation and recovery of a polycrystalline CVD diamond detector. The data suggest that the interaction mechanism of the $3.1 \mathrm{eV}$ photons in the diamond ( $E_{\text {bandgap }}=5.47 \mathrm{eV}$, thus the photons should not have enough energy to excite the diamond) is not a multi-photon process because the amplitude of the response does not increase as the square of the incident energy. This leads us to believe that photon interaction with impurities, defects (grain boundaries) in the crystal produce the signal. The first measurement results show that the detector's response clearly departs from linearity for outputs of $10 \%$ of the HV. The diamond appears not to conserve charge ( $\mathrm{S}$ departs from linearity), i.e. does not recover in time after the $1^{\text {st }}$ pulse, when the delay time is $5 \mathrm{~ns}$, but it does conserve charge in the $10 \mathrm{~ns}$ and $20 \mathrm{~ns}$ cases before saturation due to the applied voltage occurs. Therefore the detection system recovers from a saturating fist pulse after about 10 ns. Further experiments using higher-energy x-rays or electrons are planned for the future.

\section{Acknowledgments}

I would like to thank Travis Pond and Matthew Griffin from Bechtel Nevada.

\section{References}

[1] G. Schmid, R. Griffith, N. Izumi, J. Koch, R. Lerche, M. Moran, T. Phillips, R. Turner, V. Glebov, T. Sangster, and C. Stoeckl, "CVD diamond as a high bandwidth neutron detector for inertial confinement fusion diagnostics," REVIEW OF SCIENTIFIC INSTRUMENTS, vol. 74, pp. 18281831, 2003.

[2] L. Dauffy, R. Lerche, and T. Phillips, "Response of a CVD diamond detector to typical Deuterium-Tritium NIF implosions for areal density measurement," Lawrence Livermore National Laboratory, Livermore UCRL-TR-211523, 2005.

[3] L. Dauffy, R. Lerche, G. Schmid, and J. Koch, "Study of the saturation of CVD diamond detectors using laser pulses," Lawrence Livermore National Laboratory, Livermore UCRL unpublished, 2005.

[4] V. Glebov, "Progress with CVD diamond detectors for ICF neutron time-of-flight (nToF) applications," presented at the International Conference On Plasma Science (ICOPS), Monterey, CA, 2005. 